\title{
EXCHANGE CONTRIBUTIONS TO SPIN POLARIZATION IN LOW-ENERGY ELECTRON SCATTERING FROM Xe AND Hg
}

\author{
J.E. SienKIEWICZ ${ }^{a}$, S. FRITZSChE ${ }^{b}$ AND P. SYTY $^{a}$ \\ ${ }^{a}$ Department of Applied Physics and Mathematics \\ Technical University of Gdańsk, G. Narutowicza 11/12, 80-952 Gdańsk, Poland \\ ${ }^{b}$ Fachbereich Physik, Universität Kassel, 34132 Kassel, Germany
}

(Received March 14, 2000)

\begin{abstract}
The contribution of exchange interactions is investigated for spin polarization of electrons which are scattered elastically from xenon and mercury at the energy of $1.5 \mathrm{eV}$. We find that electron exchange between the bound-state and the scattered electron gives rise to a substantial spin polarization in both cases.
\end{abstract}

PACS numbers: $34.80 . \mathrm{Bm}, 31.30 . \mathrm{Jv}$

\section{Introduction}

Spin polarization of electrons scattered elastically from heavy atoms has attracted the attention of experimentalists and theoreticians for many years. A detailed account on the subject of spin polarization in the electron scattering from mercury and xenon is given in papers of Szmytkowski and Sienkiewicz $[1,2]$. Since then, the relativistic configuration interaction method has been applied also for calculating spin polarization effects in the elastic scattering of electrons from xenon [3]. Recently, moreover, Dümmler et al. [4, 5] measured the spin polarization of electrons for the elastic scattering from xenon and for the inelastic scattering from argon, krypton, and xenon. As has been pointed out by these authors, only the spin-orbit interaction is expected to contribute to the left-right asymmetry of the scattered electrons in the field of the target atom (Mott scattering). Apart from the spin-orbit interaction, however, the exchange interaction of the scattered electron with the bound-state electrons and the continuum-continuum interaction among the scattered electrons may also contribute to the spin polarization.

In this paper we investigate the effects of exchange on the spin polarization of electrons which are scattered from heavy, closed-shell atoms like xenon or mercury which has a closed $6 s^{2}$ subshell. Our aim is to demonstrate that in elastic electron scattering from the closed-shell atoms substantial contribution to 
the spin polarization arises from the exchange interactions between the scattered electron and the electrons of the target atom. In addition, we also study the discrepancy between theory and experiment which occurred for xenon at the energy of $1.5 \mathrm{eV}$ [4].

\section{Theoretical method}

The continuum orbitals are solutions of the inhomogeneous radial Dirac equations $[6,7]$ which, in atomic units, can be written as

$$
\begin{aligned}
& \left(\frac{\mathrm{d}}{\mathrm{d} r}+\frac{\kappa}{r}\right) P_{\kappa}(r)=\left\{2 c+\frac{1}{c}\left[\epsilon-V_{\mathrm{fc}}(r)-V_{\mathrm{pol}}(r)\right]\right\} Q_{\kappa}(r)+X_{Q}(r), \\
& \left(\frac{\mathrm{d}}{\mathrm{d} r}-\frac{\kappa}{r}\right) Q_{\kappa}(r)=-\frac{1}{c}\left[\epsilon-V_{\mathrm{fc}}(r)-V_{\mathrm{pol}}(r)\right] P_{\kappa}(r)-X_{P}(r) .
\end{aligned}
$$

In this coupled pair of equations, $P_{\kappa}(r)$ and $Q_{\kappa}(r)$ are the large and small radial components, and $\kappa= \pm(j+1 / 2)$ for $l=j \pm 1 / 2$ is the relativistic angular momentum quantum number, $c=137.036$ is the speed of light and $\epsilon$ is the energy of the electron. Here, $V_{\mathrm{fc}}(r)$ is the direct frozen-core potential, and $V_{\text {pol }}(r)-$ the relativistic polarization potential, while $X_{Q}(r)$ and $X_{P}(r)$, respectively, are the exchange terms for the large and small components. $V_{\text {pol }}(r)$ describes the polarization of the target atom owing to the electric field of the scattered electron; here we applied a potential in a numerical form as obtained from the relativistic polarized-orbital method [8].

The scattering equations are solved by a standard outward integration applying an algorithm developed by Sienkiewicz and Baylis [9] and starting from the boundary conditions $P_{\kappa}(0)=Q_{\kappa}(0)=0$.

The two complex scattering amplitudes $f(\vartheta)$ (the direct amplitude) and $g(\vartheta)$ (the "spin-flip" amplitude) are defined as

$$
\begin{aligned}
& f(\vartheta)=\frac{1}{2 \mathrm{i} k} \sum_{l=0}^{\infty}\left\{(l+1)\left[\exp \left(2 \mathrm{i} \delta_{l}^{+}\right)-1\right]+l\left[\exp \left(2 \mathrm{i} \delta_{l}^{-}\right)-1\right]\right\} P_{l}(\cos \vartheta), \\
& g(\vartheta)=\frac{1}{2 \mathrm{i} k} \sum_{l=0}^{\infty}\left[\exp \left(2 \mathrm{i} \delta_{l}^{-}\right)-\exp \left(2 \mathrm{i} \delta_{l}^{+}\right)\right] P_{l}^{1}(\cos \vartheta)
\end{aligned}
$$

where $\vartheta$ is the scattering angle and $P_{l}(\cos \vartheta)$ and $P_{l}^{1}(\cos \vartheta)$ are, respectively, the Legendre polynomial and the associated Legendre function $[10] ; \delta_{l}^{ \pm}$are the relativistic phase shifts. The " + " sign in $\delta_{l}^{ \pm}$refers to the solutions of Eqs. (1) and (2) with $\kappa=-l-1$ while the "-" sign refers to $\kappa=l$.

From the amplitudes (3) and (4) the spin polarization cross section is obtained from

$$
S(\vartheta)=\frac{\mathrm{i}\left(f g^{*}-f^{*} g\right)}{\sigma(\vartheta)},
$$


where $\sigma(\vartheta)=|f|^{2}+|g|^{2}$ is the differential cross section for an unpolarized incident beam [10]. Relativistic phase shifts $\delta_{l}^{ \pm}$in Eqs. (3) and (4) are calculated by comparing the numerical solutions of the Dirac-Hartree-Fock equations (1) and (2) with the asymptotic solutions at large $r$, when $r^{2} V(r) \rightarrow 0$

$$
\begin{aligned}
& \frac{P_{\kappa}(r)}{r}=j_{l}(k r) \cos \delta_{l}^{ \pm}-n_{l}(k r) \sin \delta_{l}^{ \pm}, \\
& \frac{Q_{\kappa}(r)}{r}=\alpha \sqrt{\frac{\epsilon}{\epsilon \alpha^{2}+2}}\left[j_{l}(k r) \cos \delta_{l}^{ \pm}-n_{\tilde{l}}(k r) \sin \delta_{l}^{ \pm}\right] .
\end{aligned}
$$

In these asymptotic forms, $k=\sqrt{2 \epsilon+\alpha^{2} \epsilon^{2}}$ denotes the momentum of the incident electron and $j_{l}(k r)$ and $n_{l}(k r)$ are the spherical Bessel and Neumann functions, respectively. In Eq. (7) $\tilde{l}=l \pm 1$, where the upper sign is taken for $\kappa<0$ and the lower one for $\kappa>0$. In the present work, we calculate phase shifts for all elastic channels with $l=0,1, \ldots, 6$. In Eqs. (3) and (4), the sums in the scattering amplitudes are furthermore extended to $l=50 \mathrm{using}$ an approximate non-relativistic formula of Ali and Fraser [11].

\section{Results and discussion}

Calculations have been performed for a scattering energy of $1.5 \mathrm{eV}$ which is, so far, the lowest experimental energy which was applied to xenon. We carried out two types of calculations. We first applied the full scattering equations (1) and (2). In this case both, the spin-orbit interaction as well as the exchange one between the projectile electron and the bound-state electrons, are taken into account appropriately. In the second case, we retain the exchange terms in Eqs. (1) and (2) while the spin-orbit interaction is neglected. This is obtained by using the non-relativistic limit, i.e. by multiplying the speed of light constant $c$ with a large factor, say $10^{4}$. This makes the spin-orbit interaction term and two other terms, namely the Darwin term and the relativistic mass correction term, negligible small; these three terms are of the order of $v^{2} / c^{2}$ (e.g. [12]). At low energies and subsequently small velocities of the projectile electron velocity, we do, of course, not expect other relativistic terms (apart from the spin-orbit interaction) to play any crucial role in the scattering process. The significance of the spin-orbit interaction, even at low scattering energies, is related to the slightly different scattering potentials for the electrons with different quantum numbers $\kappa$ but the same orbital quantum number $l$. Thus, in order to investigate these effects we have to retain the relativistic description of the target atom, where for instance, the $d_{3 / 2}(\kappa=2)$ and $d_{5 / 2}(\kappa=-3)$ orbitals are different. Note that for different values of $\kappa$ (e.g. $\kappa=2$ or -3 ) and the same value of $l$ (e.g. $l=2$ ) we still obtain different continuum orbitals due to slightly different exchange terms in scattering equations.

Two test calculations have been carried out to check our results, for which the spin polarization must vanish for all scattering angels. In the first test, the non-relativistic limit is taken for both, the bound-state description as well as the solving the scattering equations. In the second test, the exchange terms are omitted in nonrelativistic scattering equations, while the bound-states are obtained from relativistic calculations. Both cases were tested numerically and, as expected, did not result in any spin polarization. 


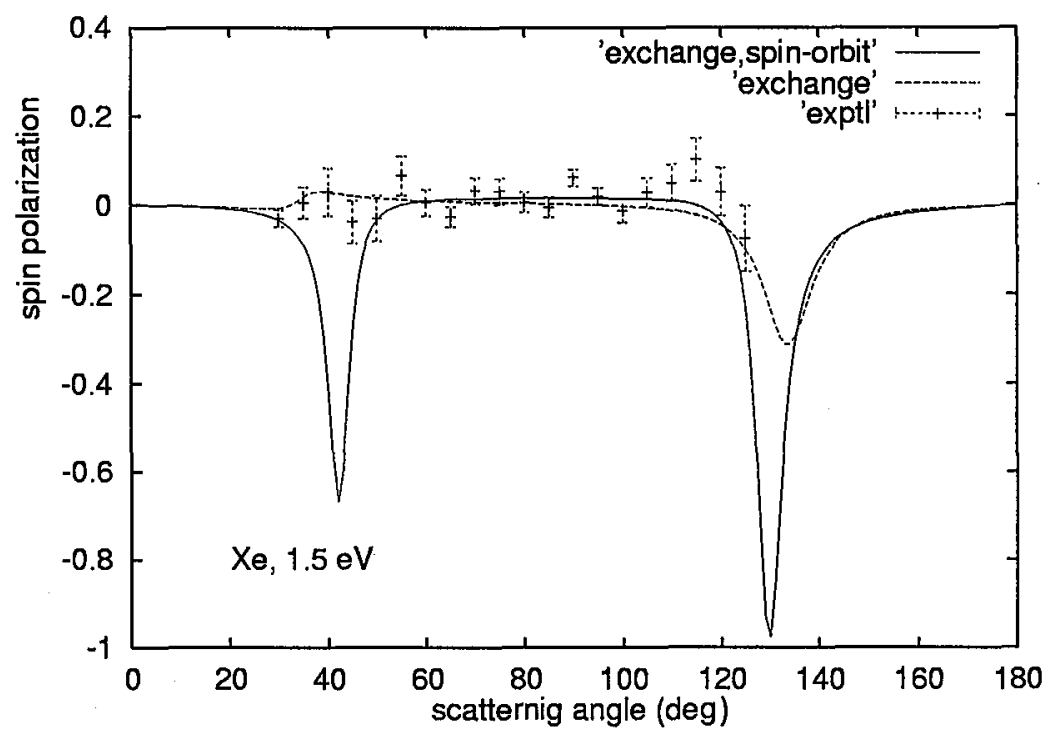

Fig. 1. Spin polarization for electron scattering from xenon at $1.5 \mathrm{eV}$. Experimental results of Dümmler et al. [4] are compared with the present theoretical results, which include the exchange and spin-orbit interactions (solid line), and only the exchange interactions (dashed line).

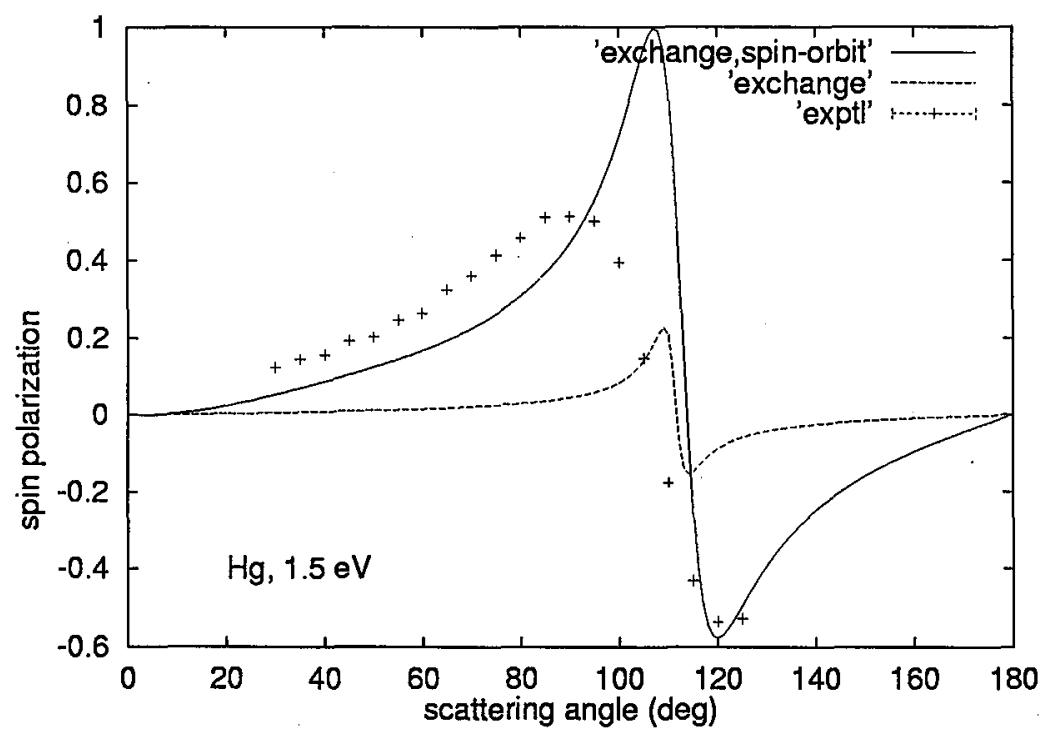

Fig. 2. Spin polarization for electron scattering from mercury at $1.5 \mathrm{eV}$. Experimental results of Dümmler et al. [5] are compared with the present theoretical results, which include the exchange and spin-orbit interactions (solid line), and only the exchange interactions (dashed line). 
Figure 1 displays our theoretical results for the elastic scattering from $\mathrm{Xe}$ at the energy of $1.5 \mathrm{eV}$ together with the experimental data of Dümmler et al. [4] which show a very small positive polarization for all scattering angles smaller than $100^{\circ}$. This observation disagrees with our present results and also with an independent theoretical study by McEachran and Stauffer [13] who obtained a similar distinct minimum at the forward angles. In fact, their results are almost identical with ours and are therefore not displayed in the figure. McEachran and Stauffer have solved the relativistic form of the Schrödinger equation with the non-relativistic polarization potential. Our calculations with the neglected spin-orbit interaction but with exchange show only the minimum at the backward scattering angles. The minimum in the spin polarization is well pronounced and shows the importance of exchange. At the forward angles, the discrepancy between theory and experiment may be caused by neglected short range correlations or by cancellation effects due to the continuum-continuum interactions. Calculations performed at the same energy for mercury also show a substantial contribution of exchange to the spin polarization at large angles (Fig. 2). In this case, however, both theoretical lines - with and without the spin-orbit interaction - show the same qualitative behaviour over the whole angular range.

\section{Conclusions}

In conclusion, the spin-flip probability due to exchange plays a quite significant role in elastic low-energy scattering from xenon. This is in contrast to earlier expectations of Dümmler et al. [4]. A comparable situation has been found in our results for mercury. Therefore, the various interactions on the spin polarization should be further investigated to understand the dominant contributions and to resolve the discrepancy between experimental and theoretical results. An advanced theoretical approach should also include the dominant parts of the continuum-continuum interaction which may cancel the influence of the spin-orbit and exchange interactions at the forward angles. To introduce continuum-continuum contributions, however, is a difficult task, in particular in relativistic calculations. We started to develop the relativistic program to include at least the interaction with polarized core charge in analogy to the nonrelativistic $N+1$ correlation method of Saha [14].

For the backward scattering, we hope that more experimental data become available to better understand the structure in the spin polarization cross sections at large angels.

This work has been supported by the Committee for Scientific Research under the grant No. 2 P03B 00912.

\section{References}

[1] R. Szmytkowski, J.E. Sienkiewicz, J. Phys. B, At. Mol. Opt. Phys. 27, 555 (1994).

[2] R. Szmytkowski, J.E. Sienkiewicz, J. Phys. B, At. Mol. Opt. Phys. 27, 2277 (1994).

[3] J.E. Sienkiewicz, S. Fritzsche, I.P. Grant, J. Phys. B, At. Mol. Opt. Phys. 28, L633 (1995). 
[4] M. Dümmler, G.F. Hanne, J: Kessler, J. Phys. B, At. Mol. Opt. Phys. 28, 2985 (1995).

[5] M. Dümmler, M. Bartsch, H. Geesmann, G.F. Hanne, J. Kessler, J. Phys. B, At. Mol. Opt. Phys. 25, 4281 (1992).

[6] I.P. Grant, Adv. Phys. 19, 74 (1970).

[7] J.P. Desclaux, Comput. Phys. Commun. 9, 31 (1975).

[8] R. Szmytkowski, Ph.D. Thesis, University of Gdan'sk, Gdańsk 1993.

[9] J.E. Sienkiewicz, W.E. Baylis, J. Phys. B, At. Mol. Opt. Phys. 20, 5145 (1987).

[10] J. Kessler, Polarized Electrons, Springer-Verlag, Berlin 1976, p. 37.

[11] M.A. Ali, P.A. Fraser, J. Phys. B, At. Mol. Opt. Phys. 10, 3091 (1977).

[12] A. Davydov, Quantum Mechanics, Pergamon Press, Oxford 1976, p. 262.

[13] R.P. McEachran, A.D. Stauffer, J. Phys. B, At. Mol. Opt. Phys. 19, 3523 (1986).

[14] H.P. Saha, Phys. Rev. Lett. 65, 2003 (1990). 